\title{
The Relationship of Nursing College Students' Stress Experiences, Eating Habits, Healthy Lifestyle, and Wellness
}

\author{
Meera Park ${ }^{1}$ and Nam Joo Je ${ }^{2 *}$ \\ Assistent Professor, Dept. nursing Changshin University, Korea \\ ${ }^{1}$ minerva32@cs.ac.kr, ${ }^{2 *}$ jnj4757@cs.ac.kr
}

\begin{abstract}
This study was conducted on nursing college students to understand stress experiences, eating habits, healthy lifestyle, level of wellness, and their relationship. The purpose of the study is to collect basic data for preparing measures to improve the wellness of nursing college students. The data were collected from 167 nursing students at $K$ University in G-do from September $15^{\text {th }}$ to September $30^{\text {th }}$, 2020. The research tool consists of 39 questions of stress experiences, 11 questions of eating habits, 7 questions of a healthy lifestyle, and 29 questions of wellness, and the collected data were analyzed using IBM SPSS WIN/25.O. The general characteristics were analyzed by frequency, mean, and standard deviation. For the difference of variables according to the general characteristics, $t$-test and one-way ANOVA were used in the analysis. The post analysis was conducted using Scheffe's test. The correlation between each variable was analyzed using Pearson's correlation. Based on the results of the study, it was found that stress experiences had an inverse correlation with a healthy lifestyle and a positive correlation with wellness. Eating habits had a positive correlation with a healthy lifestyle and wellness. A healthy lifestyle had a positive correlation with wellness. Reflecting this, to improve wellness, it is necessary to prepare a program that lowers stress experiences and improves eating habits and healthy lifestyles.
\end{abstract}

Keywords: Stress experiences, Eating habits, Healthy lifestyle, Wellness, College student

\section{Introduction}

\subsection{Significance}

College students, who are in their early adulthood, cannot be free from the anxiety and stress that comes from acquiring knowledge or information to prepare for a job or their future. In Kim's study [1], stress was found to have a negative correlation with the quality of life, and stress of college students was a factor that had a great influence on their quality of life. Therefore, it can be seen that stress has a great influence on the quality of life. In particular, nursing students suffer from a strict curriculum, heavy volume of learning, strict code of conduct, the psychological pressure of a national exam, practice in various clinical situations, and a sense of responsibility, which becomes factors in causing a lot of stress [2]. In addition, the academic stress of nursing students was found to be higher than other college students [3].

College students tend to become free from the protection and control of parents, and often experience the change of residence types (boarding house, dormitory, or living alone) without proper education on diet, due to the excessive competition for college entrance exams [4].

Article history:

Received (November 28, 2020), Review Result (January 3, 2021), Accepted (February 17, 2021) 
Inadequate eating and lifestyle habits during this period can result in health problems such as chronic diseases [5]. However, according to preceding studies on the dietary life of college students [6][7][8], the dietary life of college students had numerous problems in inadequate eating and lifestyle habits such as high rate of skipping meals, irregular mealtime, and excessive diet due to the increase in personal free time, part-time jobs, interest in appearance, and dating. Also, according to previous studies related to the lifestyle of college students [9][10][11], the smoking and drinking rates of college students were very high with some students having a high rate of binge drinking, and generally do not exercise regularly.

For modern people, the concept of health expands to a more active and holistic meaning by including all of the related elements and is called wellness. Wellness refers to the process of actively striving to change lifestyle toward optimal health, and is an effort to bring the body and mind into an optimal state which is a state of emotional, social, intellectual, physical, and spiritual harmony [12]. Anspaugh [13] defined wellness as a concept that helps the change into a desirable lifestyle and maximizes the potential of individual health for the optimal health condition that can improve the quality of life. This concept is the opposite of disease and is also expressed as a positive factor of health. With the recent increase in living standards and social interest in the quality of life, the concept of health is expanding to include not only physical health but also psychosocial and spiritual well-being.

In particular, nursing students are preliminary health care professionals who need to help maintain and improve the health of the subject in clinical practice and should be the role model of the subject with their desirable eating habits and healthy lifestyle. Stress experience, dietary behavior, and a healthy lifestyle are correlated [14], and since wellness, which is an optimal state of harmony between body and mind, is important for nursing students who provide care, we want to investigate the relationship between these variables.

Therefore, this study was attempted to identify the stress experiences, eating habits, healthy lifestyle, and wellness of nursing students and understand the relationship between these variables to use them as basic data for preparing measures to improve the wellness of nursing students.

\subsection{Objectives}

This study is aimed to understand the general characteristics of the subjects, to identify the differences in college-level stress, eating behavior, healthy lifestyle, and wellness according to the general characteristics and to grasp the correlation between each variable.

\section{Contents}

\subsection{Study design}

This study is descriptive survey research conducted to understand the relationship between the variables of stress experiences, eating habits, healthy lifestyle, and wellness of nursing students and to grasp the correlation between each variable.

\subsection{Subjects}

For the number of subjects, G* power 3.1.9 program [15] was used to calculate the minimum. Using correlation analysis, the main analysis method, with effect size .3 (medium), significance level $(\alpha) .05$, statistical power $(1-\beta) .95$, the minimum number of subjects with an appropriate sample size was 138.168 subjects were initially sampled considering the $20 \%$ dropout rate. Of 
these, 167 were used in the final analysis with the exclusion of one copy that had a poor response.

\subsection{Study tools}

This study used a structured questionnaire consisting of 92 questions in total. The questions measure general characteristics, college-level stress, eating behavior, healthy lifestyle, and wellness.

\subsubsection{General characteristics}

The general characteristics of this study were measured in a total of 6 questions referring to the previous study [16], including age, sex, height, weight, residence type, and semester rating.

\subsubsection{Stress experiences}

The stress experiences of this study were measured in a total of 39 questions tool from Yoo et al., [16]. The subdomains of stress experiences consisted of academic, interpersonal, environmental, and intrapersonal stress. The score is a 5-point Likert scale with 1 point meaning 'Not feeling stressed at all' to 5-point meaning 'Very stressful'. The reliability of the tool was Cronbach's $\alpha=.90$ at the time of development. This study's Cronbach's $\alpha=.95$.

\subsubsection{Eating habits}

The eating habits of this study were measured in a total of 11 questions tool made by Jang [17] was used. The usual eating habits were divided into 11 questions such as 'eating three meals a day regularly', 'eating balanced meals', and 'consuming milk or dairy products every day' and scored in a 5-point Likert scale with 5-point meaning 'Always' to 1 point meaning 'Never'. Of the 11 questions, 3 questions, 'Eating snacks', 'Eating fast food', and 'Doing other things while eating', were reverse-coded. The reliability of the tool was Cronbach's $\alpha=.72$. This study's Cronbach's $\alpha=.72$.

\subsubsection{Health lifestyle}

The healthy lifestyle of this study was measured in a total of 7 questions tool made by Jang [17] was used. The healthy lifestyle of this study was divided into drinking frequency, smoking, perception of weight control, the reason for weight control, exercising frequency, type of exercise, stress, type of stress, stress coping skills, and healthy lifestyle. Questions made by Jang [17] were used. The healthy lifestyle was divided into 7 questions such as 'Is healthy overall', 'Reading/watching articles or TV programs about health', and 'Taking vitamins or nutritional supplements regularly on a 5-point Likert scale with 5-point meaning 'Always' to 1 point meaning 'Never'. The reliability of the tool was Cronbach's $\alpha=.69$. This study's Cronbach's $\alpha=.68$.

\subsubsection{Wellness}

The wellness of this study was measured in a total of 29 questions tool. The Korean Wellness Scale for Adolescents (K-WSA) developed by Hong [18] was used. It consisted of 29 questions in total, is divided into 5 subdomains. The subscale was 10 questions for confidence factor (1, $\left.2,20,22,23,24,25,26^{*}, 28,29\right), 7$ questions for emotional factors $\left(13,14^{*}, 15^{*}, 16^{*}, 17^{*}, 19^{*}\right.$, $\left.27^{*}\right), 2$ questions for physical factors $\left(3^{*}, 6\right), 3$ questions for self-protection factors $(4,5,10)$, 
and 7 questions for social factors $\left(7,8,9,11,12,18^{*}, 21\right)(*=$ Recoded questions). The scale was evaluated on a 5-point scale with a higher score meaning higher wellness. The reliability of the tool was Cronbach's $\alpha=.86$ at the time of development. This study's Cronbach's $\alpha=.81$.

\subsection{Data collection period and method}

The data were collected from September $15^{\text {th }}$ to $30^{\text {th }}, 2020$ for nursing students of $\mathrm{K}$ University in G-do. To protect the subjects' autonomy and rights, the researchers explained the purpose of the study, procedures, and guarantee of anonymity and received consent from subjects who agreed to participate. Being free to withdraw the participation at free will with no penalty was notified to the participants. The average time to complete the questionnaire was 20 minutes. For personal information protection, the received data were identified by numbers and stored separately in a lockable storage file, and access was restricted to the researchers only. It was explained that the questionnaires will be stored for 3 years after the completion of the study and incinerated afterward. Also, the researchers explained that personal information and survey data of the subject related to the study will be de-identified and be used only for research purposes to guarantee confidentiality and anonymity. After the self-administered questionnaires were filled and turned in, the data of a total of 167 subjects were analyzed, excluding one questionnaire with incomplete answers.

\subsection{Data analysis}

The collected data were analyzed using IBM SPSS WIN/25.0. The general characteristics of the subjects were analyzed with frequency, average, and standard deviation. The difference in stress experiences, eating habits, healthy lifestyle, and wellness according to general characteristics were analyzed with t-test and one-way ANOVA. The post-hoc analysis was done using Scheffe's test. Pearson's Correlation was used to analyze the correlation among each variable.

\subsection{Results}

\subsubsection{Stress experiences, eating habits, healthy lifestyle, and wellness according to general characteristics}

The subjects' stress experiences, eating habits, healthy lifestyle, and wellness according to general characteristics are shown in Table 1. In stress experience, there was a statistically significant difference according to gender $(p=.042)$. Also, a statistically significant difference was found in eating habits according to gender $(\mathrm{t}=3.15, p=.002)$ and statistically significant differences depending on the type of residence $(\mathrm{F}=4.12, p=.007)$ with no statistical difference between groups in post-hoc analysis. Health lifestyle had a statistically significant difference according to gender $(\mathrm{t}=2.55, p=.011)$ and had a statistically significant difference depending on semester rating $(\mathrm{t}=-2.47, p=.014)$. Wellness had a statistically significant difference according to grades $(\mathrm{F}=3.84, p=.011)$. [Table 1].

\subsubsection{Subjects' stress experiences, eating habits, healthy lifestyle, and wellness scale}

The subjects' stress experiences, eating habits, healthy lifestyle, and wellness are shown in [Table 2]. Stress experiences had a point of 2.64 \pm 0.77 (5-point scale), eating habits $2.68 \pm 0.60$ point (5-point scale), health lifestyle $3.02 \pm 0.74$ point (5-point scale), and wellness with $3.38 \pm 0.31$ point (5-point scale). [Table 2]. 
Table 2. Subject's stress experiences, eating habits, healthy lifestyle, and wellness scale $(\mathrm{N}=167)$

\begin{tabular}{|c|c|c|c|}
\hline Variables & Mean \pm SD & Min & Max \\
\hline Stress experiences & $2.64 \pm 0.77$ & 0.74 & 4.69 \\
\hline Eating habits & $2.68 \pm 0.60$ & 1.27 & 4.09 \\
\hline Health lifestyle & $3.02 \pm 0.65$ & 1.14 & 4.71 \\
\hline Wellness & $3.38 \pm 0.31$ & 2.59 & 4.66 \\
\hline
\end{tabular}

\subsubsection{Correlation among research variables}

The correlation among stress experiences, eating habits, healthy lifestyle, and wellness of the subjects are shown in Table 3. Stress experiences had an inverse correlation with a healthy lifestyle $(\mathrm{r}=-.21, p=.006)$ and a positive correlation with wellness $(\mathrm{r}=.37, p<.001)$. Eating habits were positively correlated with a healthy lifestyle ( $\mathrm{r}=.56, p<.001)$ and wellness $(\mathrm{r}=.22, p=.003)$. Health lifestyle was positively correlated with wellness $(\mathrm{r}=.32, p<.001)$.

Table 3. Correlation among the research variables $(\mathrm{N}=167)$

\begin{tabular}{|c|c|c|c|c|}
\hline \multirow{2}{*}{ Variable } & 1 & 2 & 3 & 4 \\
\cline { 2 - 5 } & \multicolumn{4}{|c|}{$\mathrm{r}(p)$} \\
\hline 1 & 1 & & & \\
\hline 2 & $-.13(.076)$ & 1 & & 1 \\
\hline 3 & $-.21(.006)$ & $.56(<.001)$ & 1 & \\
\hline 4 & $.37(<.001)$ & $.22(.003)$ & $.32(<.001)$ & 1 \\
\hline
\end{tabular}

\section{Conclusions}

This study was conducted to understand the relationship between stress experiences, eating habits, healthy lifestyle, and wellness scale for nursing students. Stress experiences had an inverse correlation with a healthy lifestyle and a positive correlation with wellness, eating habits had positive correlations with healthy lifestyle and wellness, and a healthy lifestyle had a positive correlation with wellness. Also, stress experiences, eating habits, and a healthy lifestyle all had significant differences according to the gender of the subjects. To improve the wellness of nursing students, positive stress experiences and a healthy lifestyle are important, and providing programs to promote stress experiences and a healthy lifestyle is needed. Also, when applying for the programs, gender differences should be taken into the results and caused differences from the studies that undertook in the pre-COVID-19 situation. Therefore, to generalize the research results, replication studies to confirm the results are required. Also, additional studies consideration to effectively lower stress, establish a good eating habit and a healthy lifestyle. Since this study was targeting nursing students in one area, there is difficulty in the generalization of the results. Also, the study was conducted in pandemic situations caused by COVID-19, so environmental stress could have affected including an extended range of regions, and verifying the effectiveness of a wellness-promoting program is suggested.

\section{Acknowledgments}

This work was supported by the Changshin University Research Fund of 2020 (Changshin2020-007). 


\section{References}

[1] N. E. Kim, "The relationships among stress, resilience, and quality of life in college students," Journal of Adolescent Welfare, vol.13, no.4, pp.1-19, (2011)

[2] M. H. Jeong and M. A. Shin, "The relationship between self-esteem and satisfaction in major of nursing Students," The Journal of Korean Academic Society of Nursing Education, vol.12, no.2, pp.170-177, (2006)

[3] Y. J. Son, E. Y. Choi, and Y. A. Song, "The relationship between stress and depression in nursing college students,” Korean Journal of Stress Research, vol.18, no.4, pp.345-351, (2010)

[4] S. J. Kwon, J. H. Kang, N. J. Kim, R. J. Kim, S. Y. Kim, S. I. Kim, and G. S. Han, "Relationships between dietary behaviors with smoking, drinking situations and subjective health status of university students," Journal of Dental Hygiene Science, vol.12, no.2, pp.145-153, (2012)

[5] S. L. Lee and S. H. Lee, "Survey on health-related factors, nutrition knowledge and food habits of college students in Wonju area," Journal of Korean Community Nutrition, vol.20, no.2, pp.96-108, (2015) DOI:10.5720/kjen.2015.20.2.96

[6] B. B. Choi, "Dietary habits and behaviors of college students in the northern Gyeonggi-do region," Journal of Korean Food \& Nutrition, vol.26, no.3, pp.404-413, (2013) DOI:10.9799/ksfan.2013.26.3.404

[7] Y. S. Jun, M. K. Choi, and Y. J. Bae, "Night eating and nutrient intake status according to residence type in university students," Journal of Korean Society of Food Science and Nutrition, vol.44, no.2, pp.216-225, (2015) DOI:10.3746/jkfn.2015.44.2.216

[8] H. H. Kim, Y. Y. Jung, and Y. K. Lee, "A comparison of salty taste assessments and dietary attitudes and dietary behaviors associated with high-salt diets in four regions in Korea," Journal of Korean Community Nutrition, vol.17, pp.38-48, (2012)

[9] S. W. Park, "A review of cigarette smoking-related behaviors and health problems among university students," Korean Journal of Health Education and Promotion, vol.28, no.5, pp.35-49, (2011)

[10] B. Y. Cho, J. Y. Lee, and J. H. Young, "Behavioral factors associated with health, obesity, and subjective health status among college students," Journal of Health and Fundamental Medical Science, vol.5, no.2, pp.58-63, (2012)

[11] Y. H. Park, "Factor affecting health-related quality of life in university students: A focus on binge drinking," Journal of Lerner-Centered Curriculum and Instruction, vol.18, no,3, pp.301-318, (2018) DOI: 10.22251/jlcci.2018.18.3.301

[12] B. Hettler, "Wellness promotion a university campus: Family and community health," Journal of Health Promotion and Maintenance, vol.3, pp.77-95, (1980)

[13] D. J. Anspaugh, "Personal wellness assessments and lifestyle change," Journal of Health Education, vol.26, no.3, p.188, (1995)

[14] S. H. Cheong, Y. J. Na, E. H. Lee, and K. J. Chang, "Anthropometric measurement, dietary behaviors, healthrelated behaviors and nutrient intake according to lifestyles of college students," Journal of the Korean Society of Food Science and Nutrition, vol.36, no.12, 1560-1570, (2007) DOI: 10.3746/jkfn.2007.36.12.1560

[15] F. Faul, E. Erdfelder, A. Buchner, and A. G. Lang, "Statistical power analyses using G* power 3.1: Tests for correlation and regression analyses, behavior research methods," vol.41, no.4, pp.1149-1160, (2007) DOI: 10.3758/BRM.41.4.1149

[16] J. S. Yoo, S. J. Chang, E. K. Choi, and J. W. Park, "Development of a stress scale for Korean nursing students," Journal of Korean Academy Nursing, vol.38, no.3, pp.410-419, (2008)

[17] H. Y. Jang, "Relationship between dietary behavior, health lifestyle, and college life satisfaction according to the body shape satisfaction of university students in Jeonbuk area," M.S. thesis, Chonbuk National University, Chonbuk, (2019)

[18] D. W. Hong, "The development of wellness scale for adolescents and the effects of wellness program," Ph. D. dissertation, Kyungpook National University, Daegu, (2008) 


\section{Authors}

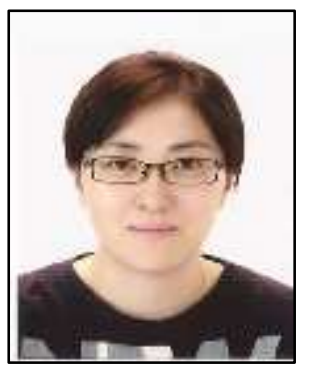

\section{Meera Park}

Assistant Professor

Department of nursing, Changshin University, Changwon

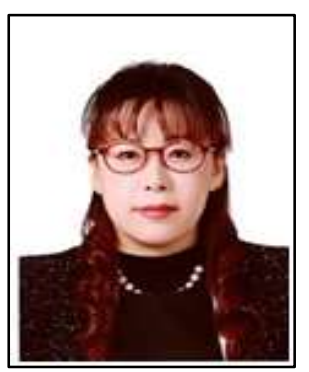

Nam Joo Je, RN, PhD

Associate Professor

Department of nursing, Changshin University, Changwon 
The Relationship of Nursing College Students' Stress Experiences, Eating Habits, Healthy Lifestyle, and Wellness

This page is empty by intention. 